\title{
Studying and Analyzing Virtualization While Transition from Classical to Virtualized Data Center
}

\author{
Amit Wadhwa \\ Assistant Professor \\ Amity University Haryana
}

\author{
Ankit Garg \\ Assistant Professor \\ Amity University Haryana
}

\begin{abstract}
This paper discusses and brings together a detailed analysis of virtualization implemented at various levels of computing infrastructure, keeping in mind and providing detailed transition process adopted for transition from a classical to virtualized data center. It focuses on emerging trends or areas related with storage virtualization. Further, its various network level virtualization based implementations has been presented and analyzed. And finally it sums up with step by step process of transition from a classical to virtualized data center.
\end{abstract}

\section{General Terms}

Virtualization, Storage Virtualization, Network Virtualization, Storage Provisioning, Virtual machine (VM)

\section{INTRODUCTION}

Virtualization is an approach with which one can pool and share IT resources so they can flexibly and automatically meet fluctuating business needs. Virtualization has its footprints in the IT industry for many years in various different forms as network, storage and server virtualization. IBM has led the way with their VM systems [2]. While moving into a cloud based environment virtualization acts as a switch or can be said as a first step towards building a cloud infrastructure. In one talks about storage part of it virtualization is the bridge between classical data center to virtualized data center. For moving to virtualized architecture firstly one has to understand the concept of virtualization and its various forms like memory, server, network and storage.

\section{CLASSIFICATION OF}

\section{VIRTUALIZATION TECHNIQUES}

Virtualization as described above is used to abstract the actual view of the computing infrastructure from user perspective and it can be implemented at various different levels of computing infrastructure. It can be further classified into various techniques discussed as under:

\subsection{Memory Virtualization}

Memory technology has changed since the evolution of concept of virtual memory. The size of memory acts as an important factor in determining the no of applications that can run over a host. With advancement in technology VMM's (i.e. virtual memory managers) have been evolved allowing multiple applications to be hosted and processed simultaneously. [8], [3] In order to implement virtual memory a separate space over hard disk is utilized acting as space used for swapping active and inactive memory pages. The access to this virtual space is controlled by VMM's.

\subsection{Server Virtualization}

It resolves the issues present in a dedicated physical server environment where one face problems of resource conflicts in a client/server environment when two or more applications running on servers have conflicting requirements. [1] These emerging issues are resolved by incorporating virtualization at server level. Introducing a virtualization layer between operating system and hardware resolves resource conflicts by isolating different applications running on different operating systems, on same machine.

\subsection{Network Virtualization}

Creating virtual network resolves the issues of managing a large network by providing feature of centralized management of devices. It is made feasible with the evolution of VLAN's (virtual local area networks), which allows users with similar access requirements grouped together in same virtual network. [3]Recent evolution to this technique is Virtual SAN or virtual fabric which improves scalability, availability and security of SAN connections by separating fibre channel services in different VSAN's (virtual storage area networks) and isolating traffic between various VSAN's.

\subsection{Storage Virtualization}

It deals with presenting a logical view of physical storage resources to various hosts over a connection. The important advantages of this kind of virtualization is increased storage utilization and easy addition and deletion of storage without affecting data availability and it also provides advantage if non-disruptive data migration across universally located data centers [3], [6]. Storage virtualization has developed its various facets in recent times like block, file and network level storage virtualization.

\section{STORAGE VIRTUALIZATION IMPLEMENTATION LEVELS}

It can be implemented at various different levels like compute, network and storage. The different levels of it describe different approach toward implementing it considering various important issues of IT infrastructure moving towards cloud computing. 


\subsection{Compute Level}

Role of it at compute level could be visualized as under:

- At this level the storage is managed and provisioned for virtual machines (VM's) running on different compute systems in a data center.

- VM's works on a hypervisor layer which enables compute system partitioning thereby acting as compute virtualization software.

- VM's are stored as set of files managed by VMFS [3] (virtual machine file system) on available memory space known to hypervisor.

- Vdisk (i.e. virtual disk) files are used by VM's to store their data thereby acting as local storage to them.

- VM's are unaware of the underlying storage technologies used and total storage available with hypervisor.

Next step or implementation level is related to storage.

\subsection{Storage Level}

One approach towards its implementation is storage provisioning, with which one could assign the storage to hosts based on various different considerations like capacity, availability and performance requirements of various applications running over it. [3] There are two approaches towards its implementation, one is "Traditional" and other one is "Virtual".
Following is the comparative analysis of both its facets:

- Traditional Provisioning: In this a group of disks are clustered together and a specific RAID level [3] is implemented over it making it as a RAID drive-set. From this drive-set specific variable sized blocks are created which ultimately gets allocated as per their requirements to hosts or users connected with that storage facility.

In this provisioning method, these fixed sized storage blocks are rigidly allocated to a host. The logical block created in this technique is referred as thick logical block because of its rigidity.

- Virtual Provisioning: In this case of provisioning storage, the blocks of storage created are not assigned physical storage at the time of their creation and final allocation to host requiring storage [3]. Physical storage is allocated to host as per the demand of the host connected over this virtual storage facility. In this architecture the physical storage is being used in a shared manner and every host is presented with more storage then its actual physical allocation. It results into more efficient storage allocation to connected hosts. The logical block created in this technique is referred as thin logical block/unit. This architecture could be better visualized with the Figure 1, given below.

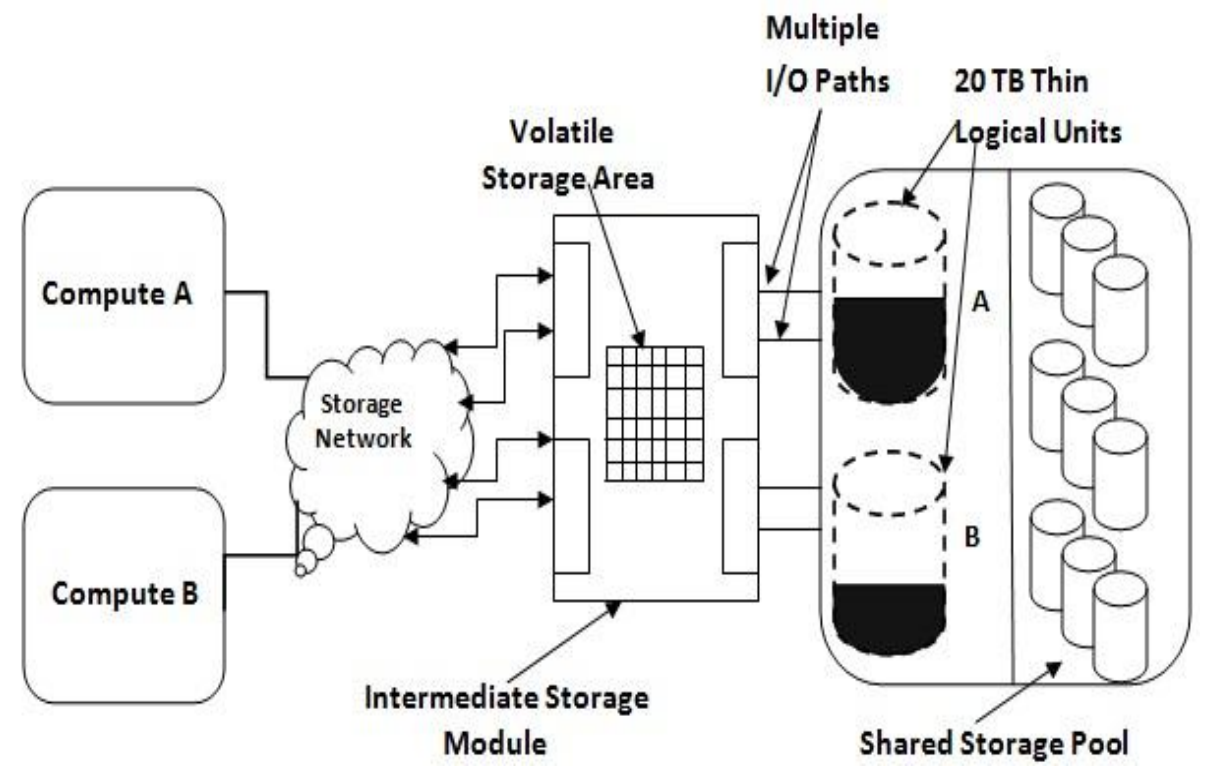

\subsection{Network Level}

At network level there are two ways of doing it considering various emerging storage technologies (i.e. SAN and NAS).

- Network based Block Level Storage Virtualization and

- Network based File Level Storage Virtualization

Figure 1: Virtual Storage Provisioning Architecture

\subsubsection{Block Level Storage Virtualization Using SAN}

In this technique instead of allocating actual physical storage to host connected over the storage area network, virtual volumes are created over a layer known as virtualization layer and allocated to host connected with the underlying 
architecture [3]. This layer is responsible for mapping of actual physical blocks of storage created over storage arrays with virtual volumes created at virtualization layer. This layer holds a shared storage pool created from varying storage devices, which is used to create needed virtual volumes. Hosts over storage area network are unaware about this abstraction of actual physical storage and the said mapping. Advantage of this type of architecture is allowing dynamic increase in storage allocations. The said architecture can be visualized with Figure 2 given here:

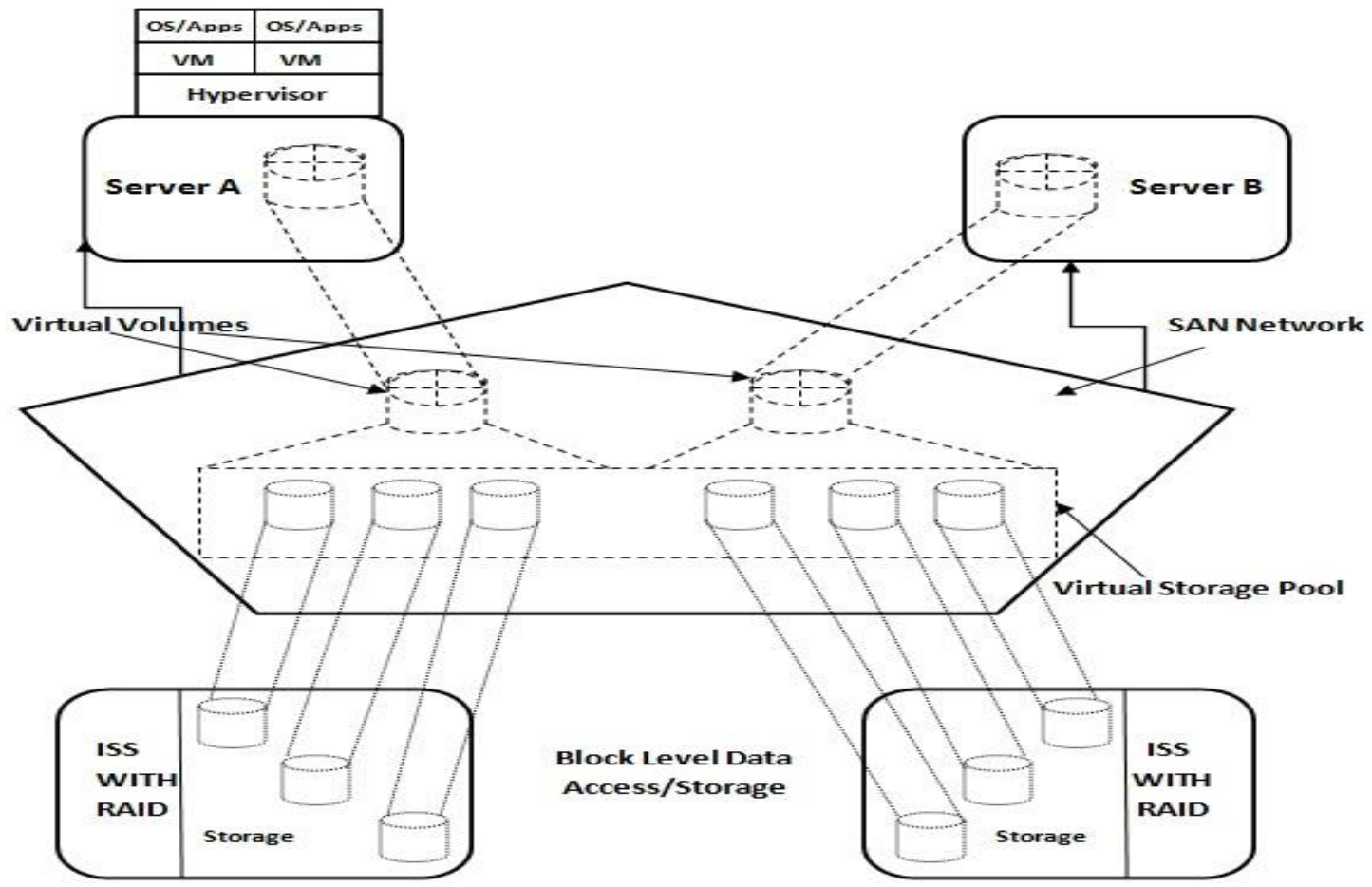

Figure 2: Block Level storage Virtualization using SAN Technology

\subsubsection{Block Level Storage Virtualization Using} SAN

In this technique of storage virtualization dependencies between location of data accessed at file level and actual data is being removed. [3] Basically, a logical pool of storage is created in this architecture at network level to remove limitation of using actual physical path to access a file. Hosts can use a logical path to get access to their data accessed in form of files. Advantage of this technique is flexibility in moving data from one location to other without hampering the data availability of end users. At the time of data movement also users can access their data uninterruptedly. This can be visualized with Figure 3 as shown here:

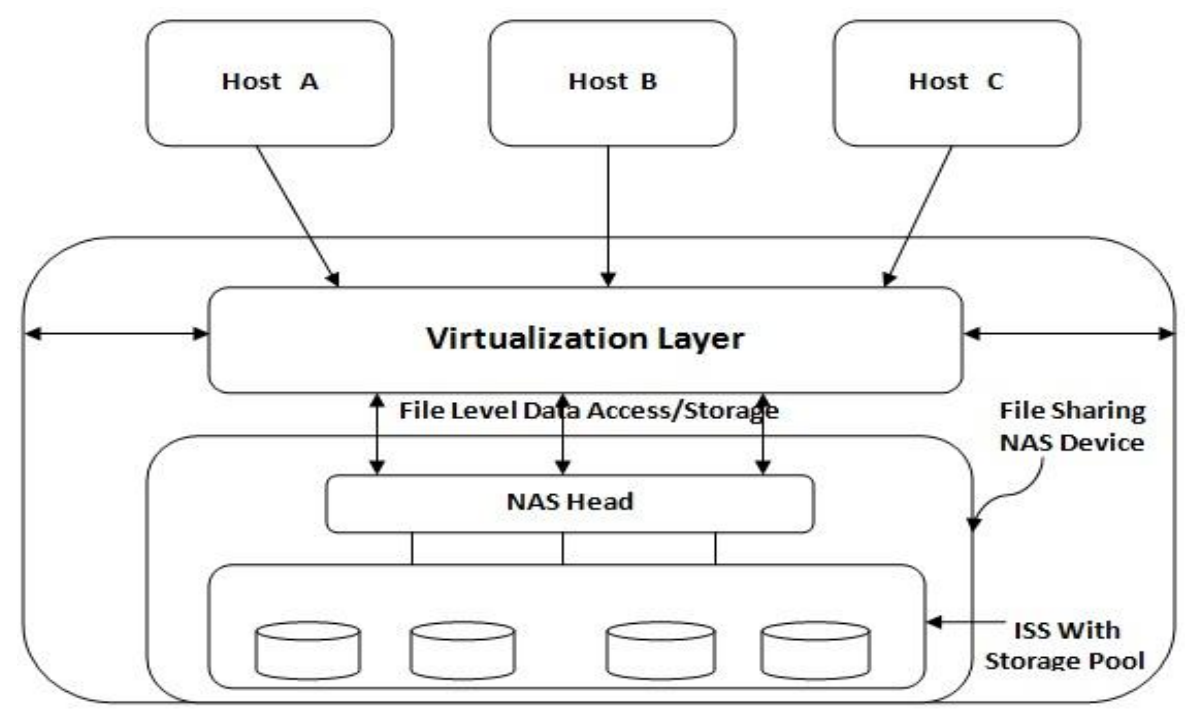

Figure 3: File Level storage Virtualization using NAS Technology 


\section{TRANSITION FROM A CLASSICAL TO VIRTUALIZED DATA CENTER}

Over a period of time various facets of virtualization has emerged in the field of IT like compute, network and storage as discussed above. [4] Each one of them is important to consider while moving into the cloud environment. So, for moving or building a cloud infrastructure one should virtualize each and every component of a "Classical DC" (CDC), i.e. compute, network and storage.

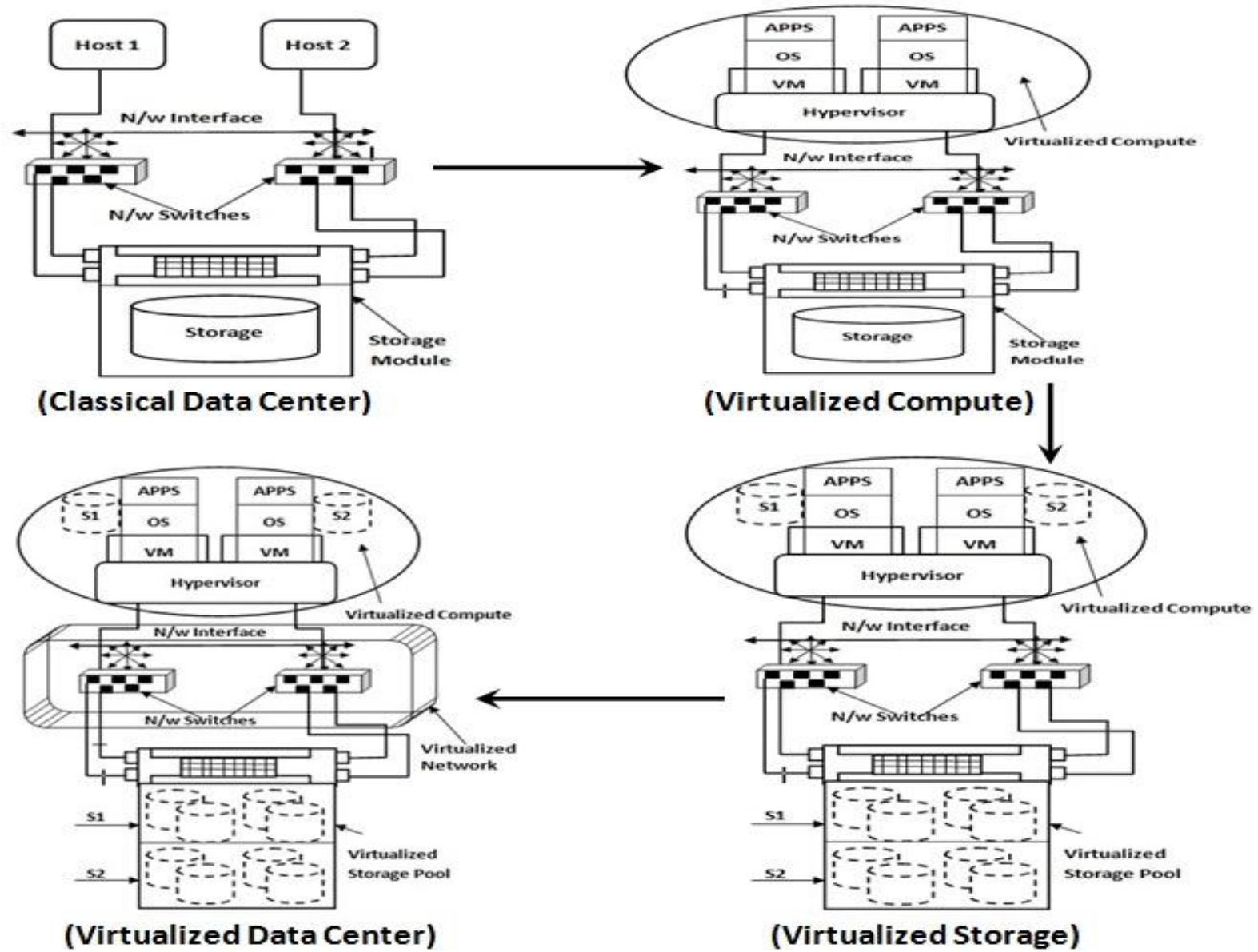

Figure 4: Moving from Classical to Virtualized Data Center

The Figure 4 above pictorially explains the step by step process of virtualizing various components of classical data center, i.e. compute, network and storage in order to make it a virtualized data center facility.

- Step 1. As the first step, a hypervisor layer is introduced in compute systems to abstract the hardware components from software layer applications and OS. Dependency or rigidity of OS is removed with introducing VMs for having different OS and applications installed over shared physical/hardware layer.

- Step 2. Second step would be to virtualize the storage area and use virtual provisioning concept to create and allocate logical units (thin LUNs [3]) to host/compute over the network. Using thin logical units in the architecture would improve the storage utilization and efficiency.

- Step 3. Next and final step is to virtualize the network layer by using either VLAN or VSAN [3] techniques to abstract their utilization and access.
The final module created after all these steps is the virtualized data center which acts as the basis for cloud computing based infrastructure.

\section{CONCLUSION}

From the above discussions, one would be able to figure out various virtualization techniques used at various places in computing infrastructure. Then storage virtualization levels that are worked upon are put forward with their differentiation. Then it brings together how virtualization is implemented at storage and network level using various storage provisioning techniques and its network based implementations respectively. Finally after understanding virtualization and its implementations at various compute infrastructure levels one would be able to perform the transition from a classical to virtualized data center thereby virtualizing every component of a classical data center as explained with Figure 4 above. 


\section{REFERENCES}

[1] Agrawal, D.,El Abbadi, A., Wang, S., Secure and Privacy- Preserving Data Services in the Cloud: A Data Centric View, Presented at the 38th International Conference on Very Large Data Bases, August 27th 31st 2012, Istanbul, Turkey

[2] Gurav, U. and Shaikh, R. 2010. "Virtualization- A key feature of cloud computing" International Conference and Workshop on Emerging Trends in Technology (ICWET 2010) - TCET, Mumbai, India

[3] Gnanasundaram, S. and Shrivastava, A. Information Storage and Management. USA: Wiley, 2013. Print

[4] Wadhwa, A. and kamra, R. "Analysing Security Threats and Solutions during Transition to Cloud Environment" presented in National conference on Innovations in Computing and Information Technology (Cognition 2015), Feb 25-26, 2015, Gurgaon, India

[5] Chraibi, M., Harroud, H. and Maach, A. "Classification of Security Issues and Solutions in Cloud Environments", International conference based on Adhoc generalized Wireless Sensor and networks, by ACM, at Vienna, Austria, Dec, 2013

[6] VMware, Inc. white paper. Virtualization: Architectural Considerations and Other Evaluation Criteria. http://www.vmware.com/pdf/virtualization_consideratio ns. pdf.

[7] Lombardi, F. and Di Pietro, R. "Secure virtualization for cloud computing" Journal of Network and Computer Applications, June 2010

[8] Raj, G. and Setia, S. "Effective Cost Mechanism for Cloudlet Retransmission and Prioritized VM Scheduling Mechanism over Broker Virtual Machine
Communication Framework". International Journal on Cloud Computing: Services and Architecture (IJCCSA),Vol.2, No.3, June 2012

[9] Bourguiba, M. et al "Improving Network I/O Virtualization for Cloud Computing". IEEE TRANSACTIONS ON PARALLEL AND DISTRIBUTED SYSTEMS, Vol 25, No. 3, March 2014

[10] Wang, J., Wright, K. and Gopalan, K. "XenLoop: A Transparent High Performance Inter-VM Network LoopBack," Proc. ACM Symp. High Performance Parallel and Distributed Computing (HPDC '08), 2008

[11] Gamage, S. et al. "Opportunistic Flooding to Improve TCP Transmit Performance in Virtualized Clouds," Proc Second ACM Symp. Cloud Computing (SOCC '11), 2011

[12] Zhang, X. et al. "XenSocket: A High-Throughput Interdomain Transport for Virtual Machines," Proc. ACM/IFIP/USENIX Int'l Conf. Middleware (Middleware '07), 2007

[13] Calheiros, R. N. et al. "CloudSim: A Novel Framework for Modeling and Simulation of Cloud Computing Infrastructures and Services”. published in IEEE, 2009

[14] Rutkowska, J. "Security Challenges in Virtualized Environments", white paper, Nordic Virtualization Forum, October 2007

[15] Christodorescu, M. et al. "Cloud Security is not (just) Virtualization Security”, (CCSW'09), Nov.13, 2009, Chicago, Illinois, USA

[16] Yu, A., Qin, Y. and Wang, D. "Obtaining the Integrity of Your Virtual Machine in the Cloud" In CloudCom '11, pages 213-222, 2011 